Trauma Berufskrankh 2005 · 7[Suppl 1]:S130-S133 DOI 10.1007/s10039-004-0944-z

Online publiziert: 11. September 2004

๑) Springer Medizin Verlag 2004
P. Kujath $\cdot$ H. Esnaashari

Universitätsklinikum Schleswig-Holstein, Campus Lübeck

\title{
Weichgewebsinfektionen und Fasziitis
}

H aut- und Weichgewebsinfektionen sind häufig. Die Inzidenz liegt zwischen 5 und 10\% im chirurgischen Krankengut. Das Spektrum der Erkrankungen reicht von der harmlosen Furunkulose und begrenzten Phlegmonen bis zu lebensbedrohlichen Infektionen wie die nekrotisierende Fasziitis, die unbehandelt rasch zum Tode führen. Der englische Mikrobiologe Kingston [8] hat 1990 für den chirurgischen Bereich eine praktikable Einteilung vorgelegt, die auf der Rasanz der Entwicklung der Infektionen beruht (• Tabelle 1). Dabei ist es wichtig für den Therapeuten, das Ausmaß einer Infektion korrekt einzuschätzen. Dies gilt ebenso für die schweren Weichteilinfektionen als auch für postoperative Wundinfektionen, die oft verschleiert verlaufen können und bei denen die Entscheidung zur operativen Revision nur anhand mehrerer klinischer Kriterien gefällt werden kann. Im angloamerikanischen Schrifttum wird häufig der Ausdruck „komplizierte Haut-WeichgewebsInfektionen“ gebraucht. Die Definition beruht auf Kriterien der amerikanischen Food and Drug Administration (• Tabelle 2).

\section{Ätiologie}

Die Entstehung von Haut und Weichgewebsinfektionen erfolgt am häufigsten durch Verletzung der Hautbarriere. Zumeist besteht eine direkte Abhängigkeit vom Ausmaß der Traumatisierung und dem dabei erfolgten Verschmutzungsgrad. Andererseits können auch kleine Bagatellverletzungen wie intramuskuläre Injektionen zu desaströsen Verläufen führen [3, 4].
Es wurde immer wieder versucht, durch Scoresysteme Weichgewebsinfektionen untereinander vergleichbar $\mathrm{zu}$ machen. Bislang gibt es kein etabliertes Scoresystem. Die Erklärung dafür liegt in den un-

Tabelle 1

Einteilung der Weichteilinfektionen entsprechend der Dringlichkeit der chirurgischen Versorgung

1. Leichte Infektionen, die konservativ behandelt werden können („slowly progressive infection“): - Furunkulose

- Begrenzte Phlegome - Erysipel

2. Infektionen, die einer dringlichen chirurgischen Versorgung bedürfen:

- Panaritium

- Abszess (Sonderformen: Karbunkel, Hidradenitis suppurativa,

Acne inversa, Spritzenabszesse, periproktitischer Abszess)

- Phlegmonen

- Eitrige Bursitiden

3. Schwere nekrotisierende lebensbedrohliche Weichteilinfektionen, die ohne chirurgische Intervention tödlich verlaufen („,rapidly progressive infection"):

- Myonekrose des Gasbrandes

- Nekrotisierende Fasziitis

- Fournier-Gangrän

- Nekrotisierende Mischinfektion

Kingston 1990

Die 3. Gruppe wird derzeit im amerikanischen Schrifttum zur Gruppe der NSTI (,,necrotizing soft tissue infections") zusammengefasst
Tabelle 2

Kriterien der amerikanischen FDA

für komplizierte Haut-

und Weichteilinfektionen

1. Die Infektion erfordert eine größere chirurgische Intervention (z. B. Débridement von devitalisiertem Gewebe, Abszessdrainage, Entfernung von Fremdkörpern, die die Infektion unterhalten, operativer Faszienschnitt)

2. Der Infektionsprozess erfasst nachweislich auch tiefergelegene Weichteilgewebe, die Faszie- und/oder die Muskelschicht

3. Eine schwere Grunderkrankung, welche das Ansprechen der Therapie erschwert, u. a.

- Diabetes mellitus

- Bakteriämie

- Phlegmone mit Beteiligung von mehr als 3\% der Körperoberfläche - Kortikoidtherapie (über 7,5 mg/Tag Prednisolonäquivalent)

- Neutropenie, Granulozytenzahl $<500 / \mu l$

- Leberzirrhose (Child-Klassifikation B oder C)

-Verbrennung (über $10 \%$ der Körperoberfläche)

- Strahlentherapie lokal oder systemisch

- Anamnestisch bekannter Alkoholismus (über 6 Monate)

- Organtransplantation

- Mangelernährung

-Immunsuppressive Therapie

Ferner ist es von Bedeutung, ob die Infektion im Krankenhaus (nosokomial) entstanden ist oder ambulant erworben wurde 
Tabelle 3

Ausprägungsformen des

clostridialen Gasbrandes

1. Syndrom der ",simple contamination" - C. sporogens, C. bifermentans, C. putrificum und grampositive Kokken - Oberflächliche, grün-bläuliche, z. T. schwärzliche Wunde - Gute Prognose

2. "Anaerob cellulitis" - Subkutane Gasbildung, lokalisiert oder diffus

- Kaum Allgemeinreaktion

- Schmutzige Wunde mit fauligem Geruch

3. Anaerobe Myonekrose,,/Gasbrand “ - Akuter, dramatischer Verlauf mit Allgemeinsymptomen - Die Muskulatur ist betroffen, grünlich, schwärzlich, manchmal bräunliche Verfärbung, stark wechselnd

Weinstein und Barza 1973

terschiedlichen Formen und der Virulenz der auslösenden Erreger. So können einzelne Stämme von Streptokokken oder Staphylococcus aureus eine extreme Virulenz aufweisen, die in seltenen Fällen zu schnellen tödlichen Verläufen führen kann $[1,13]$. Andererseits können Wunden durch Staphylococcus aureus kontaminiert werden, ohne dass eine begleitende schwere Infektion auftritt. Ausschlaggebend für die Virulenz der Erreger sind bestimmte Virulenzfaktoren, bei denen man im wesentlichen 5 funktionelle Klassen unterscheidet:
1. Adhäsine,
2. Aggressine,
3. Invasine,
4. Impedine,
5. Moduline.

Diese Virulenzfaktoren können extrem unterschiedlich ausgeprägt sein. Wichtig für den Therapeuten zu wissen ist, dass Virulenzfaktoren und antibiotisches Resistenzverhalten in keiner Weise miteinander zusammenhängen und in Korrelation zu bringen sind.

Bei den Adhäsinen handelt es sich meist um fibronektinbindende Proteine, die es dem Erreger möglich machen, sich an Geweben festzusetzen. Zu den Aggressinen ge-

\section{Tabelle 4}

Kriterien zur Diagnose eines "toxic shock-like syndrome" (STSS)

I. Isolierung von Streptokokken der

A: Gruppe A (Streptococcus pyogenes) Entnommen von einer normalerweise sterilen Körperregion oder -flüssigkeit (z. B. Blut, Liquor, Pleura- oder Abdominalflüssigkeit, Gewebebiopsie, chirurgische Wunde etc.)

B: Entnommen von einer nichtsterilen Körperregion oder -flüssigkeit (z. B. Rachenabstrich, Sputum, Vagina, oberflächliche Hautläsion)

\section{Klinische Zeichen}

A: Hypotonie: systolischer Blutdruck $\leq 90 \mathrm{mmHg}$

B: $\quad \geq 2$ der folgenden Kriterien: - Nierenversagen: Kreatinin $\geq 177 \mu \mathrm{mol} / \mathrm{l}$ ( $\geq 2 \mathrm{mg} / \mathrm{dl}$ )

- Bei vorbestehender Niereninsuffizienz eine $\geq 2$ fache Erhöhung des Kreatininwertes

- Koagulopathie: Thrombozytenzahl $\leq 100.000 / \mu \mathrm{l}\left(\leq 100.000 / \mathrm{mm}^{3}\right)$ oder disseminierte intravasale Gerinnung (DIG)

- Leberversagen: GOT,GPT oder Bilirubin auf $\geq 2$ faches der Norm erhöht - Bei vorbestehender Lebererkrankung eine $\geq \mathbf{2 f a c h e ~ E r h o ̈ h u n g ~ d e r ~}$ Werte

- Lungenversagen (ARDS)

- Generalisiertes, erythematöses Hautexanthem, evtl. mit Blasenbildung

-Weichgewebsnekrosen, nekrotisierende Fasziitis, Myositis, Gangrän

Broll et al. 1998

Als gesichert gilt die Diagnose TSLS, wenn die Kriterien IA und II (A und B) erfüllt sind, als wahrscheinlich, wenn die Kriterien IB und II (A und B) - bei Ausschluss anderer Krankheitsursachen) - erfüllt sind

hören meist wenig spezifische zytolytische Faktoren („Spreading-Faktoren“), Streptolysine, Hyaluronidasen oder die Streptokinase, die auch zu therapeutischen Zwecken eingesetzt wird. Um sich innerhalb von Geweben ausbreiten zu können, benötigen Bakterien ferner Invasine, denen es möglich ist, durch Epithel- und Endothelzellen hindurch zu dringen. Die Impedine führen zu einer Herabsetzung der Abwehrreaktion des Wirtes. Sie greifen meist bei
Tabelle 5

Diagnosekriterien der

nekrotisierenden Fasziitis

Extensive Nekrose der Faszie mit

Ausdehnung auf die angrenzende Haut

Mittlere bis schwere Systemintoxikation mit vermindertem mentalen Status

Fehlen einer primären Muskelbeteiligung

Fehlen von Clostridien im Wundabstrich

Fehlen eines ursächlichen

Gefäßverschlusses

Leukozyteninfiltration, fokale Nekrosen der Faszie und des umgebenden Gewebes sowie mikrovaskuläre Thrombosen bei der histologischen Untersuchung

Fisher, Conway 1979

den Makrophagen und Leukozyten an und führen dort zu einer Hemmung der Phagozytose. Durch die Moduline werden Erreger derartig verändert, dass sie von den $\mathrm{Ab}$ wehrmechanismen des Wirtes nicht mehr richtig erkannt werden und sich somit über Jahre und Jahrzehnte in einem Organismus etablieren können (Tuberkulose).

Die am meisten nachgewiesenen Erreger von Haut- und Weichgewebsinfektionen sind grampositive Erreger mit den häufigsten Vertretern Staphylococcus aureus und den Streptokokken. Auch Anaerobier können an Weichgewebsinfektionen beteiligt sein (Clostridium perfringens als ursprünglicher Erreger des Gasbrandes). Gramnegative Erreger wie Klebsiellen und Pseudomonaden sind besonders in Krankenhäusern als persistierende nosokomiale Erreger gefürchtet.

\section{Die klinischen Erkrankungen}

\section{Gasbrand}

1. Stadium. Schon Hippokrates hat Krankheitsbilder beschrieben, die auf die Gasbrandinfektion zutreffen. Die Erkrankung ist selten und gehört zu den meldepflichtigen Erkrankungen. 1997 wurden im Bundesgebiet 150 Fälle registriert. Weinstein [17] hat 3 Stadien der Erkrankung charakterisiert, dabei handelt es sich um differente Krankheitsbilder, die nicht in einander übergehen (• Tabelle 3). Das 1. Stadium (,simple contamination") zeigt einen grünschwärzlichen 
Trauma Berufskrankh

$2005 \cdot 7$ [Suppl 1]:S130-S133

DOI 10.1007/s10039-004-0944-z

c) Springer Medizin Verlag 2004

\section{P. Kujath $\cdot H$. Esnaashari}

\section{Weichgewebsinfektionen und Fasziitis}

\section{Zusammenfassung}

Die schweren, nekrotisierenden Weichgewebsinfektionen sind selten. Wegen der schlechten Prognose muss die Diagnostik frühzeitig einsetzen und durch eine chirurgische Freilegung verifiziert werden. Die Therapie nekrotisierender Weichgewebsinfektionen erfolgt in einem radikalen Débridement, dem ein programmiertes Redébridement folgt. Die Prognose in Bezug auf die Letalität korreliert mit der Zeitdauer zwischen Beginn der Infektion und der chirurgischen Intervention.

\section{Schlüsselwörter}

Weichgewebsinfektion - Débridement . Prognose - Gasbrand · Nekrotisierende Fasziitis

\section{Soft tissue infections and fasciitis}

\begin{abstract}
Severe necrotizing soft tissue infections are rare. The poor prognosis makes early diagnosis necessary, verified by surgery. The treatment of necrotizing soft tissue infections consists of radical débridement, followed by repeat débridement. Prognosis with regard to lethality correlates with the time between the onset of infection and the surgical intervention.
\end{abstract}

\section{Keywords}

Soft tissue infection · Débridement . Prognosis · Gas gangrene · Necrotizing fasciitis
Wundbelag oberflächlicher Wunden. Meist lässt sich Clostridium sporogines, Clostridium bifermentans und Clostridium putrificum nachweisen. Diese Erkrankungsform bedarf einer offenen Wundbehandlung wie bei jeder verschmutzten Wunde.

2. Stadium. Für das 2. Stadium der anaeroben Zellulitis wird eine Inkubationszeit von 3-4 Tagen angegeben. Meist zeigt sich ein scharf begrenzter, lokalisierter „Gasabszess" bei einer schmutzig stinkenden Wunde, besonders im Subkutangewebe. Bei dieser Erkrankung ist eine umgehende Spaltung des Subkutangewebes erforderlich. Vor allem ist chirurgisch zu klären, ob die Muskulatur mit befallen ist. Liegt nur eine lokalisierte subkutane gasbildende Infektion mit Clostridium perfringens vor, so muss eine lokalisierte Wundbehandlung vorgenommen werden, was zu einer raschen Ausheilung der Infektion führt.

Zur antibiotischen Behandlung wird die Kombination von Penicillin G und Metronidazol empfohlen. Man muss jedoch davon ausgehen, dass die meisten verletzungsbedingten Gasbrandfälle polymikrobielle Infektionen sind. Insofern sollten Antibiotika mit einem breiteren Spektrum eingesetzt werden $[5,6]$.

3. Stadium. Die 3. Erkrankungsform, die Myonekrose, ist der eigentliche Gasbrand. Es handelt sich um eine Infektion quergestreifter Muskulatur mit einem charakteristischen Krankheitsbild. Nach akutem Beginn mit kurzer Inkubationszeit zeigen sich die Anfangssymptome mit schwerer Reduktion des Allgemeinzustandes und zerebraler Dysfunktion. Die Muskulatur ist von zerfließlicher Struktur, blutet wenig, und bei Druck auf die Muskulatur lassen sich Luftblasen abpressen. Eine Eiterbildung ist nicht vorhanden. Im Spätstadium kommt es zur Hämolyse mit Ikterus. Der Tod folgt im septischen Schock. Auffallend ist der oft stechende aasähnliche Geruch. Die Diagnose wird neben dem klinischen Bild durch das mikrobiologische Präparat verifiziert. Jedes mikrobiologische Institut ist in der Lage, innerhalb von 20 min durch eine Gramfärbung den grampositiven Erreger nachzuweisen.

Therapie. Im Stadium der Myonekrose sind abladierende Maßnahmen zur Lebensrettung unumgänglich. Aufgrund der Rasanz der Ausbreitung der Infektion sollte der Zeitpunkt zur Ablation nicht zu lange hinausgezögert werden. Die hyperbare Oxygenation hat adjuvanten Charakter, sie sollte niemals im Stadium der Myonekrose primär als singuläre Therapie eingesetzt werden [7a].

\section{Die toxischen Schocksyndrome}

Todd veröffentlichte 1978 ein Krankheitsbild von Weichgewebsinfektionen mit einer schweren Symptomatik, dass durch Staphylokokken verursacht wurde. In den 198oer Jahren wurde eine ähnliche Krankheitsbeschreibung auch auf Streptokokken zurückgeführt [18]. Seitdem werden schwere Wundinfektionen durch Streptokokken bzw. Staphylokokken „streptococcal toxic shocklike syndrome“ bzw „staphylococcal toxic shock syndrome" genannt. Die Diagnose wird dann gestellt, wenn die entsprechenden Kriterien erfüllt sind (• Tabelle 4). Am häufigsten werden diese Schocksyndrome durch Wunden verursacht, durch die virulente Streptokokken oder Staphylokokken in den Organismus eindringen. Die Therapie besteht in einem Wunddébridement und einer entsprechenden antibiotischen Therapie und einer intensivmedizinischen Behandlung mit Organersatztherapie.

\section{Nekrotisierende Fasziitis}

Diese Erkrankung ist durch eine rasche Progredienz mit sich rasch ausbreitenden Nekrosen der Faszienstrukturen gekennzeichnet. Besonders in der vorantibiotischen Ära gab es in Kriegslazaretten nach Verwundung schwere Epidemien mit extremer Letalität. Fisher [7] definierte 6 entscheidende Diagnosekriterien (• Tabelle 5). Die Inzidenz der Erkrankung wird auf 3-5: 100 .ooo pro Jahr in Nordamerika und Europa angegeben. Vom mikrobiologischen Standpunkt werden 2 Formen unterschieden: Typ 1 beschreibt eine synergistisch wirkende anaerob-aerobe Mischinfektion, beim Typ 2 steht die Infektion mit Streptokokken im Vordergrund.

Symptome. Leitsymptom der Erkrankung ist der extreme Schmerz. Dieser Schmerz lässt sich zu Beginn der Erkrankung mit dem klinischen Erscheinungsbild nicht immer in Einklang bringen („pain out of proportion“; [9]). Es zeigt sich ein unscharf be- 


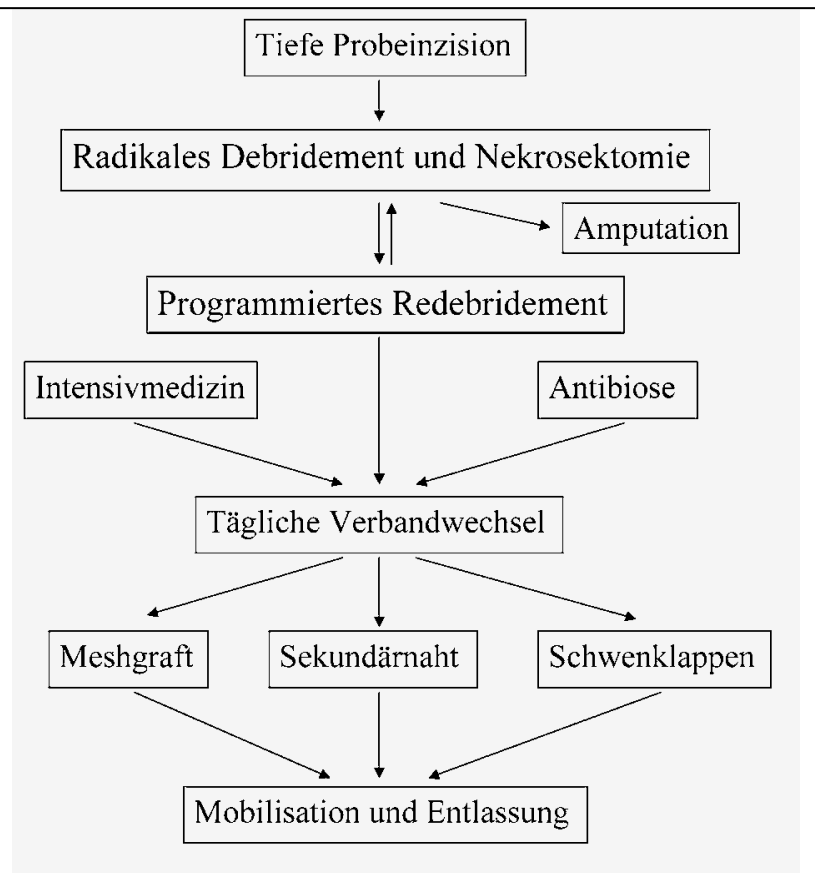

Interessenkonflikt: Der korrespondierende Autor versichert, dass keine Verbindungen mit einer Firma, deren Produkt in dem Artikel genannt ist, oder einer Firma, die ein Konkurrenzprodukt vertreibt, bestehen.

\section{Literatur}

1. Bisno AL, Stevens DL (1996) Streptococcal infections of skin and soft tissue. N Engl J Med 334: 240 245

2. Broll R, Eckmann C, Kujath P, Bruch HP (1998) Streptococcal toxic shock-like syndrome. Chirurg 69: 806-812

3. Eckmann C, Kujath P, Benecke P, Husstedt W-D (1997) Die nekrotisierende Fasziitis der Vulva. Geburtsh Frauenheilkd 57: 18-23

4. Eckmann C, Kujath P, Shekarriz H, Staubach KH (1997) Clostridiale Myonekrose als Folge intramuskulärer Injektionen - Beschreibung dreier fataler Verläufe. Langenbecks Arch ChirSuppl II (Kongreßband): 553-555

Abb. $1<$ Therapie der nekrotisierenden Fasziitis grenztes Erythem, dass später in eine livide, landkartenartige Hautverfärbung mit zentralen Nekrosen übergeht. Entsprechend dem Ausmaß der Erkrankung bestehen Allgemeinsymptome wie eine zerebrale Dysregulation und Fieber mit gelegentlichem Schüttelfrost. Die Lymphknoten sind nur selten geschwollen. In der Sonographie zeigt sich ein echoarmer Saum als Flüssigkeitsstruktur zwischen Faszie und Subkutangewebe. Eine Computertomographie oder ein MRT bringen nur selten weitere diagnostische Informationen. Bei dringendem Verdacht auf eine nekrotisierende Fasziitis sollte eine Probeinzision bis zur Faszie vorgenommen werden. Klinisch lässt sich dann die Fasziitis durch Zerfall der Faszienstrukturen und auffallende Thrombosen kleinerer Gefäße gut nachweisen. Bewiesen wird die Erkrankung durch den histologischen Befund.

Therapie. Die Therapie der Wahl ist ein frühzeitiges und radikales chirurgisches Débridement. Eine nicht ausreichende Exzision der nekrotischen Bezirke führt zu einer hohen Letalität. Je nach Ausmaß der Erkrankung sollte ein programmiertes Débridement vorgenommen werden (- Abb. 1). Ziel des Redébridements ist es, die weitere Ausbreitung der Erkrankung zu verhindern und weitere Nekrosebezirke zu exzidieren. Das Redébridement sollte so lange fortgesetzt werden, bis die Infektion beherrscht ist. Während dieser Zeit ist der Patient meist intensivpflichtig.
Die weitere Behandlung offener Wunden sollte nach einem standardisierten Therapieplan erfolgen, der sich an den Phasen der Wundheilung orientiert. Nach Aufsprießen von Granulationen sollte die Wunde durch ein plastisches Verfahren wie Meshgraft, Sekundärnaht oder Schwenklappen gedeckt werden. Zwischenzeitlich kann auch ein Vakuumverband angelegt werden. Dies sollte jedoch erst nach Beherrschung der Infektion vorgenommen werden. Da nach Beherrschung der Infektion auch ein Verschluss der Wunden gut möglich ist, sollte der Vakuumverband nicht zu lange eingesetzt werden. Die nekrotisierende Fasziitis bedarf einer frühen antibiotischen Therapie, empfohlen wird der Einsatz eines $\beta$-Lactam-geschützten Penicillins [15]. Durch Anwendung der hyperbaren Oxygenation konnte bisher kein Vorteil in der Therapie der nekrotisierenden Fasziitis belegt werden. Die Prognose ist nach wie vor vom Ausmaß der Infektion und vom Zeitpunkt der operativen Revision abhängig. Sie liegt in unterschiedlichen Kollektiven zwischen 30 und $60 \%$ $[16,12,11]$.

\section{Korrespondierender Autor Prof. Dr. P. Kujath}

Universitätsklinikum Schleswig-Holstein, Campus Lübeck,

Ratzeburger Allee 160, 23538 Lübeck E-Mail: kujath-luebeck@t-online.de
5. Erttmann M, Havemann D (1992) Behandlung des Gasödems. Unfallchirurg 95: 471-476

6. Erttmann M, Hobrecht R, Havemann D (1992) Ist Penicillin $\mathrm{G}$ das Mittel der Wahl beim Gasödem? Zentralbl Chir 117: 509-514

7. Fisher JE, Conway ML, Takeshita RT, Sandoval MR (1979) Necrotizing fasciitis. JAMA 241: 803-806

7a. Germonpré P (1995) Evaluation of antimicrobials combined with hyper-baric oxygen in a mouse model of clostridial myonecrosis. The Journal of Trauma, Injury, Infection, and Critical Care 38: 462

8. Kingston D, Seal DV (1990) Current hypotheses on synergistic microbial gangrene. Br J Surg 77: 260 264

9. Kujath P, Eckmann C (1998) Die nekrotisierende Fasziitis und schwere Weichteilinfektionen durch Gruppe-A-Streptokokken. Dtsch Ärztebl 95 (8): B 347-353

10. Marck KW, Den Hollander H, Grond JK, Veenendaal D (1996) Survival after necrotizing streptococcal myositis: a matter of hours. Eur J Surg 162: 981983

11. McHenry CR (1995) Determinants of mortality for necrotizing soft-tissue infections. Ann Surg 221: 558-565

12. Rouse TM (1982) Necrotizing fasciitis. A preventable disaster. Surgery 92: 765

13. Schwartz B, Facklam RB, Breimann RF (1994) Changing epidemiology of group $A$ streptococcal infections in the USA. Lancet 336: 1167-1171

14. Scully RE, Mark EJ, Mc Neely WF, Mc Neely BU (1995) Weekly clinico-pathological exercises. Case 21-1995. N Engl J Med 333: 113-119

15. Vogel F (2004) Empfehlungen zur kalkulierten parenteralen Initialtherapie bakterieller Erkrankungen bei Erwachsenen. Chemother J 13: 46-106

16. Voros D, Pissiotis C (1993) Role of early and extensive surgery in the treatment of severe necrotizing soft tissue infections. Br J Surg 80: 1190-1191

17. Weinstein L, Barza MA (1973) Gas gangrene. N Engl J Med 289: 1129-1131

18. Willoughby R, Greenberg RN (1983) The toxic shock syndrome and streptococcal pyrogenic exotoxins. Ann Intern Med 98: 559 